\title{
Investigation of Current Situation of Learning Motivation, Social Anxiety and Loneliness of the Left-behind Children in Rural Primary School
}

\author{
Biyun Zhang ${ }^{1}$, and Ming $\mathrm{Xu}^{2}$ \\ ${ }^{1}$ School of Education, Hubei University of Science and Technology, 437100 Xianning Hubei, China \\ ${ }^{2}$ Senior High School of Southern Hubei, 437100 Xianning Hubei, China
}

\begin{abstract}
Objective: To understand the situation of learning motivation, social anxiety and loneliness of the left-behind children. Method: Selecting three rural primary schools in Xian'an District of Xianning City to investigate left-behind situation, learning motivation, social anxiety and loneliness of pupils in Grades 4 to 6 in rural primary school in Xian'an District by the use of the MAAT-I-A which is revised by Zhou Bucheng, the Social Anxiety Scale for Children (SASC) and the Children's Loneliness Scale (CLS). Results: (1) The learning motivation of the leftbehind children in rural primary school is in a slightly higher medium level. Social anxiety is significantly higher than normal level in Chinese city, and the level of loneliness of about $1 / 5$ of the left-behind children is relatively high. (2) The score of learning motivation, social anxiety and loneliness of the left-behind children in the level of knowledge learning has significant grade differences, without significant gender differences. (3) The level of learning motivation, social anxiety and loneliness of the left-behind children is slightly higher than that of non-left-behind children, but both differences are not significant.
\end{abstract}

Keywords. left-behind children; pupils; learning motivation; social anxiety; loneliness

\section{Problems-posing}

With the rapid social transformation and urbanization, population migration and flow are gradually becoming frequent, and more and more rural workers leave their birthplace and move toward cities as migrant workers. Thus, their children become rural left-behind children. The so-called "left-behind children" refer to those children who could not live together with their parents because parents or one parent flows to other areas, and leaves their children in place of domicile.

Due to lack of parental care, the left-behind children are lack of correct guidance and assistance by the parents in the outlook on life and moral values in the growth process, and their emotion is in a state of "family hunger", thus easily causing learning problems and a 
variety of psychological problems, of which the insufficient learning motivation, social anxiety, loneliness and other issues are prominent.

The existing research on the leaning motivation of the left-behind children is rare, especially the lack of empirical research, and some of views raised are lack of strong data support. The research of the current situation and characteristics of the social anxiety and loneliness of this special group of left-behind children is insufficient, and the research of this group of pupils is few.

Therefore, through field survey and psychological measurement, based on the understanding of the general problems in the growth and education of the left-behind children in rural primary school, the research attempts to investigate the characteristics of the learning motivation, social anxiety and loneliness of the left-behind children, and reveal more about inherent characteristics of psychological development of the left-behind children.

\section{Methods}

\subsection{Research object}

The research randomly chooses 329 pupils in Grades 4 to 6 in three rural primary schools in Xian'an District of Xianning City for questionnaire survey, obtaining 321 pieces of valid questionnaires, of which there are 211 left-behind children, with the left-behind rate of $65.73 \%$. In the valid questionnaires, there are 186 boys and 134 girls; 147 pupils in Grade 4 , 152 pupils in Grade 5 and 21 pupils in Grade 6; 106 non-left-behind children, 92 left-behind children with one migrant parent; 119 left-behind children with both migrant parents. Among the left-behind children, there are 128 boys and 83 girls; 100 pupils in Grade 4, 98 pupils in Grade 5 and 13 pupils in Grade 6.

\subsection{Research tools}

\subsubsection{MAAT-IA}

MAAT "Success Motivation" Scale I-A "Knowledge Learning Scene" proposed by Zhou Bucheng is used to measure intrinsic motivation level of learning activities of rural pupils. Split-half reliability of the whole test in different tested groups is between $0.83-0.89$, while the test-retest reliability is between $0.79-0.86$. It has a good validity and it is suitable for students in Grade 4 to Senior Grade 3, which is a more mature learning motivation scale at present. Because of the characteristics of the tested itself and the needs of research, only the knowledge learning scene with comprehensive achievement motivation dimension is selected to measure internal learning motivation of rural pupils.

\subsubsection{Social Anxiety Scale for children (SASC)}

Social Anxiety Scale for children (SASC) developed by La Greca, et al is adopted. Such scale is suitable for children in primary school. There are a total of 10 projects with the existing domestic generic version. It scores by three levels ( 0 : never; 1 : sometimes; 2 : always), and the score of total scale is from 0 to 20 . The higher the total score is, the higher the degree of social anxiety is. The reliability and validity of the scale is good. Li Fei, Su Linyan and Jin Yu established Chinese Urban Norm of Social Anxiety Scale for Children in 2006. In the country, there is no social anxiety norm for rural children. Therefore, according to the boundary 
methods of Li Fei, Su Linyan, et al, $90 \%$ percentile ( $\mathrm{P}_{90}$ is 10 points in the research) is deemed as a diagnosis boundary to differentiate whether children have anxiety disorder.

\subsubsection{Children's Loneliness Scale (CLS)}

Children's Loneliness Scale (CLS) developed by Asher et al. (1984) and revised by Wang Xiangdong, et al. is adopted. Such scale is mainly used to assess degree of loneliness and social dissatisfaction of children in Grades 3 to 6 . There are a total of 24 projects. The higher the total score is, the stronger the loneliness is. The reliability and validity of the scale are good. According to the scale layered approach proposed by Cassidy, the score of Children's Loneliness Scale (CLS) can be layered. 80\% percentile ( $\mathrm{P}_{80}$ is 40 points in the research) is deemed as a critical value to distinguish the level of children's loneliness.

\subsection{Research program}

Under coordination and organization of the related teachers, the members in the research group act as main testers to carry out testing in the classroom in class time, give standard guidance, and recycle questionnaires on the spot. SPSS 17.0 software can be adopted for data processing and statistical analysis of the collected data.

\section{Conclusions}

\subsection{Current situation of learning motivation, social anxiety and loneliness of the left-behind children in rural primary school}

The total score of learning motivation of left-behind children in the research $(\mathrm{M} \pm \mathrm{SD})$ is $(27.64 \pm 3.491)$, which is significantly higher than the median $24(\mathrm{t}=14.219, \mathrm{p}<0.001)$, that is, the learning motivation of the left-behind children in rural primary school in the research is in a slightly higher medium level;

Social anxiety of the left-behind children $(M \pm S D)$ is $(5.63 \pm 3.421)$, which is significantly higher than Chinese Urban Norm $(3.48 \pm 3.47)(t=8.811, p<0.001)$, but significantly lower than the demarcation score and the median $10(\mathrm{t}=-17.875, \mathrm{p}<0.001)$; it indicates that the social intercourse emotion of the left-behind children in rural primary school in the research is more favorable on the whole, which does not reach the level of social anxiety disorder, but is higher than the average level of Chinese city;

Loneliness of the left-behind children is 33.25 , which is significantly lower than the demarcation score, 40 points $(\mathrm{t}=-8.879, \mathrm{p}<0.001)$, which indicates that the social intercourse emotion of the left-behind children in rural primary school in the research is more favorable on the whole;

From the perspective of the detection rate, most of children do not reach social anxiety disorder and high level of loneliness, but a small part of the children $(10.2 \%)$ still reach the level of social anxiety disorder, and a part of children $(22.5 \%)$ reach a high level of loneliness (see table 2). It indicates that the social intercourse emotion of the left-behind children in rural primary school in Xianning City is more favorable on the whole, but still has some problems.

\subsection{Demographic differences of learning motivation, social anxiety and lone- liness of the left-behind children in rural primary school}




\subsubsection{Differences of learning motivation, social anxiety and loneliness between the left-behind children and non-left-behind children in rural primary school}

The independent sample $t$ test is given to the differences of learning motivation, social anxiety and loneliness between the left-behind children and non-left-behind children in rural primary school. The results show that the score of learning motivation, social anxiety and loneliness of the left-behind children in rural primary school is higher than that of the non-left-behind children, but with insignificant differences (see Table 1).

Table 1. Comparison of differences of learning motivation, social anxiety and loneliness between the left-behind children and non-left-behind children in rural primary school

\begin{tabular}{lllll}
\hline & Left-behind children & Non-left-behind children & $\mathrm{t}$ & Sig.(both sides) \\
\hline $\begin{array}{l}\text { Total score of learning } \\
\text { motivation }\end{array}$ & $27.64 \pm 3.491(\mathrm{n}=186)$ & $27.11 \pm 3.895(\mathrm{n}=92)$ & -1.148 & 0.252 \\
Total score of social anxiety & $5.63 \pm 3.421(\mathrm{n}=196)$ & $5.30 \pm 3.138(\mathrm{n}=100)$ & -0.813 & 0.417 \\
Total score of loneliness & $33.25 \pm 10.138(\mathrm{n}=178)$ & $31.51 \pm 9.334(\mathrm{n}=89)$ & -1.362 & 0.174 \\
\hline
\end{tabular}

The epidemiological research of the detection rate of social anxiety and loneliness of rural pupils (in case of social anxiety disorder $\geq 10$ points, it may exist social anxiety disorder; in case of loneliness $\geq 40$ points, the level of loneliness is high) shows that, the left-behind children with anxiety account for $10.2 \%$, while the non-left-behind children with anxiety account for $11.0 \%$, both of which have no significant differences; the leftbehind children with high loneliness account for $22.5 \%$, while the non-left-behind children with high loneliness account for $20.2 \%$, both of which have no significant differences, but the number of the left-behind children with high loneliness is slightly higher than that of the non-left-behind children (see Table 2).

Table 2. Distribution characteristics of social anxiety and loneliness of children in rural primary school [n(\%)]

\begin{tabular}{lllll}
\hline Variables & & $\begin{array}{l}\text { Occurrence frequency } \\
\text { (frequency) }\end{array}$ & $\chi^{2}$ & P \\
\hline Occurrence rate of social anxiety & non-left-behind (n=100) & $11(11.0 \%)$ & 0.045 & 0.832 \\
& left-behind (n=196) & $20(10.2 \%)$ & & \\
& all(n=296) & $31(10.47 \%)$ & & \\
Occurrence rate of high loneliness & non-left-behind (n=89) & $18(20.2 \%)$ & & 0.176 \\
& left-behind (n=178) & $40(22.5 \%)$ & & \\
& all (n=267) & $58(21.72 \%)$ & & \\
\hline
\end{tabular}

\subsubsection{Gender differences of learning motivation, social anxiety and loneliness of the left-behind children in rural primary school}

The independent sample t test of learning motivation, social anxiety and loneliness of the left-behind children in rural primary school shows that the gender differences of the leftbehind children in rural primary school are insignificant in the score of learning motivation, social anxiety and loneliness in level of the knowledge learning. 
Table 3. Test results of gender differences $t$ of learning motivation, social anxiety and loneliness of the left-behind children in rural primary school

\begin{tabular}{lllll}
\hline & Male $(\mathrm{M} \pm \mathrm{SD})$ & Female $(\mathrm{M} \pm \mathrm{SD})$ & $\mathrm{t}$ & Sig.(both sides) \\
\hline Total score of learning motivation & $27.36 \pm 3.42(\mathrm{n}=114)$ & $28.08 \pm 3.575(\mathrm{n}=72)$ & -1.380 & 0.169 \\
Total score of social anxiety & $5.67 \pm 3.460(\mathrm{n}=120)$ & $5.58 \pm 3.379(\mathrm{n}=76)$ & 0.174 & 0.862 \\
Total score of loneliness & $34.31 \pm 10.683(\mathrm{n}=109)$ & $31.58 \pm 9.035(\mathrm{n}=69)$ & 1.762 & 0.080 \\
\hline
\end{tabular}

\subsubsection{Grade differences of learning motivation, social anxiety and loneliness of the left-behind children in rural primary school}

One-way analysis of variance and post multiple comparisons of learning motivation, social anxiety and loneliness of the left-behind children in rural primary school show that, the grade differences of learning motivation, social anxiety and loneliness of the left-behind children in rural primary school are significant; the post multiple comparison shows that the level of the learning motivation of the left-behind children in Grade 6 is the lowest, which is significantly lower than that of the Grade 4 and Grade 5, but there is no significant differences between the Grade 4 and Grade 5; the level of social anxiety of the left-behind children in Grade 6 is the highest, which is significantly higher than that of the Grade 4 and Grade 5, but the Grade 4 is slightly higher than the Grade 5 and without reaching a significant level; the level of loneliness of the left-behind children in Grade 5 is the lowest, which is significantly lower than that of the Grade 4 and Grade 6 , but there is no significant differences between the Grade 4 and Grade 6 (see Table 4).

Table 4. Comparative results of gender differences of learning motivation, social anxiety and loneliness of the left-behind children in rural primary school

\begin{tabular}{lllllll}
\hline & $\begin{array}{l}\text { Grade 4 } \\
(\mathrm{M} \pm \mathrm{SD})(1)\end{array}$ & $\begin{array}{l}\text { Grade 5 } \\
(\mathrm{M} \pm \mathrm{SD})(2)\end{array}$ & $\begin{array}{l}\text { Grade 6 } \\
(\mathrm{M} \pm \mathrm{SD})(3)\end{array}$ & $\mathrm{F}$ & $\mathrm{P}$ & Multiple comparison \\
\hline Total score of learning & $27.97 \pm 3.128$ & $27.66 \pm 3.497$ & $24.82 \pm 5.076$ & 4.118 & $0.018^{* *}$ & $(3)<(2)^{*} ;(3)<(1)^{* *}$ \\
motivation & $(\mathrm{n}=89)$ & $(\mathrm{n}=86)$ & $(\mathrm{n}=11)$ & & & \\
Total score of social & $\begin{array}{l}5.85 \pm 3.766 \\
(\mathrm{n}=94)\end{array}$ & $\begin{array}{l}5.04 \pm 2.654 \\
(\mathrm{n}=89)\end{array}$ & $\begin{array}{l}8.08 \pm 4.310 \\
(\mathrm{n}=13)\end{array}$ & 5.023 & $0.007^{* *}$ & $(3)>(2)^{* *} ;(3)>(1)^{*}$ \\
anxiety & $\begin{array}{l}34.78 \pm 10.823 \\
\text { Total score of loneliness }\end{array}$ & $\begin{array}{l}31.29 \pm 9.624 \\
(\mathrm{n}=78)\end{array}$ & $\begin{array}{l}37.23 \pm 6.340 \\
(\mathrm{n}=87)\end{array}$ & 3.628 & $0.029^{*}$ & $(2)<(3)^{*} ;(2)<(1)^{*}$ \\
& $(\mathrm{n}=13)$ & & & \\
\hline
\end{tabular}

(Note: ${ }^{* * *}$ represents $p<0.001,{ }^{* *}$ represents $p<0.01,{ }^{*}$ represents $p<0.05$, the same below)

\section{Analysis and discussion}

\subsection{Discussion of the current situation of learning motivation, social anxiety and loneliness of the left-behind children in rural primary school}

The research finds that in the knowledge learning scene, the learning motivation of the leftbehind children in rural primary school is in a slightly higher medium level, which may be related to more emphasis on academic performance in rural areas.

For the social anxiety and loneliness of the left-behind children in the research, an overall situation of the score and the detection rate shows that the social intercourse emotion of the left-behind children in rural primary school in Xianning City is more favorable on the whole, without reaching social anxiety disorder and high levels of loneliness, but still exists some problems. It verifies a viewpoint that the left-behind 
children are prone to have some problems in learning motivation and social intercourse emotion.

\subsection{Differential analysis of learning motivation, social anxiety and loneliness of the left-behind children in rural primary school}

\subsubsection{Differential analysis of learning motivation, social anxiety and loneliness be- tween the left-behind children and the non-left-behind children in rural primary school}

The research shows that the total score of learning motivation, social anxiety and loneliness of the left-behind children in rural primary school is higher than that of the non-left-behind children, but both of which have no significant differences, and the detection rate also does not have significant differences from the perspective of epidemiology. It is inconsistent with the research expectations and research results of the majority. In general, compared with those non-left-behind children, the left-behind children with stronger social anxiety and loneliness are more likely to have learning and emotional problems, and be lack of learning motivation, poor academic performance, poor peer relationships, and avoid interpersonal communication. It may be related to the particularity of the samples selected in the research. The factors to affect learning motivation and social intercourse emotion of the children are interacted from multiple aspects. The parents' migration and lack of parental care is not the sole determinant to affect its development, which works by the subject of children. The impact caused by lack of parental care is likely to be offset by other factors. In the research, the left-behind rate in rural primary schools is nearly $2 / 3$, and the phenomenon of left-behind children is relatively common. The left-behind children and non-left-behind children substantially live in a similar environment. The non-left-behind children in rural areas are accompanied by their parents, but their parents in rural areas generally put more emphasis on material life and academic achievement and neglect deep emotional exchange with their children. The rural students with poor emotion processing and ability to regulate and control generally have stronger learning motivation, and are lack of confidence and not good at interpersonal communication, resulting in no significant differences between the left-behind children and non-left-behind children. In addition, it may because a variety of problems will occur in the growth process of children, and these problems are not necessarily unique to the left-behind children, which are just more prominent for the left-behind children. Whether there are significant differences of learning motivation, social anxiety and loneliness between the left-behind children and the non-leftbehind children in rural primary school in each area remain to be further researched.

\subsubsection{Differential analysis of learning motivation of the left-behind children in rural primary school}

The research finds out that, the learning motivation of the left-behind children in rural primary school in the level of knowledge learning does not have significant gender differences, which is consistent with the research of pupils done by many researchers.

The rural students generally put more emphasis on academic achievement. No matter boys or girls among the left-behind children, they have stronger learning motivation, and desire to achieve excellent achievement in school work. The learning motivation of the leftbehind children in rural primary school has a certain individual difference, but without gender differences. 
Variance analysis shows that the learning motivation of the left-behind children in rural primary school has significant grade differences. The learning motivation of the left-behind children in Grade 6 is significantly lower than that of the Grade 4 and Grade 5, while the learning motivation of the left-behind children in Grade 4 is slightly higher than that of the Grade 5, but without reaching a significant level. For the characteristics of grade differences of learning motivation of pupils, the researchers also have disagreement. The situation of the left-behind children in rural areas may be different from the pure research of pupils. The majority of researches on the learning motivation of primary and secondary school students show that, the learning motivation of students may present a downward trend with the increase of the grade. Viewing from the mean value, the research also presents such trend. In addition, the level of learning motivation in Grade 6 in the research is significantly lower than the level of motivation in Grade 4 and Grade 5, which is consistent with the research of pupils in Grade 4 to 6 done by Ma Qiong (2014) and Zhang Min (2005). With the increase of grade, the students gradually reduce their feeling of freshness for many things. However, with the gradually increasing academic difficulty and more and more arduous learning tasks, the students in Grade 6 also face the pressure of entering a high school, and the society, schools and families put too much emphasis on academic achievement. This phenomenon is particularly evident in rural areas, resulting in lesser interest in knowledge, and increasing school-weary emotion with the increase of grade, so that the learning motivation of pupils in Grade 6 is significantly lower than that of the Grade 4 and Grade 5.

\subsubsection{Differential analysis of social anxiety of the left-behind children in rural prima- ry school}

Frank and Hymel believe that, the anxiety level of boys and girls are the same, but the boys are not much more willing to admit it than girls.

The left-behind children in rural primary school in the research do not have significant gender differences; the level of social anxiety of the left-behind children in Grade 6 is the highest, which is significantly higher than that of the Grade 4 and Grade 5; Grade 4 is slightly higher than Grade 5, but without reaching a significant level.

The research of Zhou Zongkui, et al. also shows that the level of anxiety of boys is the same with that of girls; the research of Zhang Shun and Wang Liangfeng, et al. shows that, the gender of the left-behind children is also not significant in the main effect of social anxiety. No matter primary school boys or girls, they put their main vigor on school work. However, this era puts an increasing emphasis on the importance of social intercourse; social skills of pupils are not yet mature; without companion of their parents, the leftbehind children look forward to interacting with others to gradually meet the needs of social intercourse. Therefore, the level of social anxiety of boy and girls does not have significant differences.

For the grade differences, the social anxiety of lower grade students is higher than that of senior grade students, presenting a downward trend with the grade, which is consistent with the research results of the original scale. In the research, the social anxiety of students in Grade 6 is higher than that of Grade 4, presenting a recovery trend in Grade 6, which is consistent with the research result of Zhou Zongkui, et al. Perhaps, the students in Grade 6 face the pressure of admission test without time to consider the needs of social intercourse. However, the preadolescent juveniles increase their self-consciousness, and are more concerned about some of the self-expression factors related to social anxiety than young children, resulting in a significant increase of social anxiety. 


\subsubsection{Differential analysis of loneliness of the left-behind children in rural primary school}

In numerous researches of children's loneliness, there is a majority of discussion on the grade and gender differences of loneliness, but without final conclusion. Some scholars believe that the consistency of comparative research findings should be combined with specific research background. The research of Zhang Qian (2013) finds that the loneliness of the non-left-behind children in rural areas has gender differences, and the boys are significantly higher than girls; the level of loneliness of the left-behind children does not have gender differences. Through $t$ test, the research finds that among the left-behind children in rural primary school, the loneliness of boys is slightly higher than that of girls but without significant gender differences, which is consistent with the research of the leftbehind children done by Duan Baojun. Gender differences of the loneliness of the non-leftbehind children are mainly caused by the following reasons: Girls are more gregarious than boys and better at language expression and communication skills or social culture; however, such impact is weakened in the family of left-behind children with the migration of one parent or both parents, with parent-child separation and lack of parental care. The leftbehind boys and girls are relatively introverted and cautious, lack of confidence, and not good at talking nor interpersonal communicating, so the loneliness felt by both sides does not have significant differences.

The research of common children by the original scale shows that the loneliness of students does not have grade differences. The research of Liu Junsheng and Zhou Ying, et al. (2013) finds that, the loneliness of pupils in Grades 2 to 5 presents a curved downward trend, the progressive decreasing rate gradually slows down, and the initial level and growth rate have significant individual differences, that is, with the increase of grade, the loneliness presents a downward trend. The previous numerous researches also show that, the loneliness of children presents a downward trend with the increase of grade. With the increase of grade, the cognitive abilities and social skills of the children gradually increase; the ability to cope with loneliness, process and regulate emotion is getting stronger; the loneliness will be getting lower.

The research finds that the loneliness of the left-behind children in Grade 6 presents a recovery trend, perhaps, because the children in Grade 6 are in pre-adolescence, the level of self-consciousness increases rapidly, and these children desire to be accepted and adored by other students. In addition, they face the entrance exams with great academic pressure, parent migration, lack of parental care and emotional exchange, so they have stronger loneliness. However, Grade 4 is a crucial turning point of the physical and mental development of the children, so that the level of loneliness of the left-behind children in Grade 4 and Grade 6 does not have significant differences.

\section{Conclusions}

(1) The learning motivation of the left-behind children in the rural primary school is in a slightly higher medium level. Social anxiety is significantly higher than norm level in Chinese city, and the level of loneliness of about $1 / 5$ of the left-behind children is relatively high. (2) The score of learning motivation, social anxiety and loneliness of the left-behind children in the level of knowledge learning has significant grade differences without significant gender differences. (3) The level of learning motivation, social anxiety and loneliness of the left-behind children is slightly higher than that of non-left-behind children, but both differences are not significant. 


\section{Acknowledgement}

Fund Project: Special Research Project of Key Subject Construction in Hubei Province; Education Discipline Construction; Funding Project of Key Education Discipline Construction in Hubei Institute of Science and Technology (Project Number: hkj010).

\section{References}

1. Liu Xiaoling. 2015. Relationship research of learning motivation level of pupils, peer interaction and academic achievement, Shanghai Education and Research, 4.

2. Zhang Min, Lei Kaichun \& Wang Zhenyong. 2005. Structural analysis of learning motivation of pupils in Grade 4 to 6. Psychological Science. 28(1).

3. Zhou Zongkui \& Fan Cuiying. 2001. Research of social anxiety and loneliness of primary school children. Psychological Science. 24(4): 442-444.

4. Ma Hong. 1993. Social Anxiety Scale for Children. Journal of Chinese Mental Health, 13: 248-249.

5. Liu Junsheng, Zhou Ying \& Li Dan. 2013. Development track of middle-late loneliness in childhood: Analysis of latent variable growth model, Journal of Psychology.

6. Du Lingli, Fan Zhiguang, Wei Xin, \& Li Ying. 2012. Research of aggressive behavior and loneliness of the left-behind children in cities, Journal of Chinese Health Psychology.

7. Yang Wanqiu, Gao Wanhong \& Liu Yi, 2014. Comparative research of the left-behind children and non- left-behind children in rural minority areas, Educational Forum.

8. Yang Jiping \& Gao Ling. 2005. Present situation research of pupils' learning psychology and teacher-student relationship. Education Research, (1): 63-64.

9. Zhang Qian. 2013. Comparative research of mental health and behavioral problems of the left-behind children and non-left-behind children in rural areas in Shandong Province, Shandong University [Master's thesis].

10. Hu Lirong \& Hu Birong. 2008. Comparison with the social anxiety and loneliness of pupils, China School Health .8.

11. Ren Ning \& Shen Li, 2008.Current situation research of the loneliness of the leftbehind children in rural primary school, Journal of Chinese Health Psychology.

12. Jane Fuping. 2011. Research of information processing features of the rural left-behind children with different level of loneliness for the lack of parental care, $\mathrm{PhD}$ thesis of Southwest University, 6. 